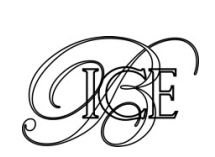

Judith Arnal Martinez*

Antonio José Bravo Álvarez**

\title{
UN BREVE ESTUDIO DE LA LEY 5/2015 DE FOMENTO DE LA FINANCIACIÓN EMPRESARIAL
}

Recientemente, se ha aprobado la Ley 5/2015, de 27 de abril, de fomento de la financiación empresarial. Esta ley busca tanto resolver algunos problemas de la financiación bancaria a las pymes como, sobre todo, sentar las bases para fortalecer las fuentes de financiación corporativa directa (no bancaria) en España. En este artículo repasaremos brevemente las principales medidas que incluye como la «Información Financiera-PYME», la revisión del marco de titulizaciones o la regulación del crowdfunding de tipo financiero.

Palabras clave: pyme, titulización, valores, crowdfunding.

Clasificación JEL: G18, G21, G23, K22.

\section{Introducción}

El objetivo clásico del sistema financiero es la asignación eficiente de los fondos, permitiendo su traspaso desde las entidades superavitarias (ahorradores) hasta las entidades deficitarias (inversores).

En España, la reciente crisis financiera se trasladó rápidamente al conjunto de la economía real, impactando de forma muy negativa en las tasas de crecimiento del PIB y el empleo. Esta importante interrelación entre las economías real y financiera puede explicarse por dos fenómenos. En primer lugar, en el sistema financiero español, como en gran medida en el europeo, el sector bancario ha tenido tradicionalmente un papel protagonista, intermediando entre ahorradores y prestatarios. Esta fuerte dependencia empresarial con respecto

* Subdirección General de Asuntos Económicos y Financieros de la Unión Europea.

** Subdirección General de Legislación y Política Financiera.

Versión de julio de 2015. al crédito bancario hace que, frente a una crisis del sistema bancario como la vivida en España, el acceso de las empresas a sus fuentes de financiación habituales se vea claramente limitado. En segundo lugar, la dependencia de la financiación bancaria es aún más acusada en el caso de las pequeñas y medianas empresas (pymes), para las que más del 70 por 100 de la financiación proviene del canal bancario. A este hecho hay que sumar la importante participación de las pymes en la economía española, que suponen más del 99 por 100 del tejido empresarial.

La crisis del sistema financiero español ha motivado la adopción de un importante número de medidas adoptadas de forma sucesiva, que podrían clasificarse en dos grandes grupos. El primer grupo estuvo destinado a la estabilización del conjunto del sistema bancario español y se implementó en estrecha relación con el programa de asistencia financiera para la recapitalización de las entidades financieras españolas. Concluido el programa de asistencia financiera de forma exitosa $\triangle$ 
en diciembre de 2013, se comenzó con la preparación de una segunda ola de reformas, tendentes a fomentar el acceso de las empresas no financieras españolas a fuentes de financiación no bancarias. En esta segunda línea se ha aprobado recientemente la Ley $5 / 2015$, de 27 de abril, de fomento de la financiación empresarial ${ }^{1}$, cuyas principales medidas repasaremos brevemente en este artículo².

En un primer momento, nos detendremos en las mejoras de la financiación bancaria a las pymes. Si bien el objetivo principal de la norma consiste en facilitar el acceso de las pymes a la financiación alternativa, no hay que olvidar el papel preponderante que el sector bancario seguirá jugando en la economía española. Por ello, esta ley trata de facilitar el acceso a la financiación bancaria a través de medidas como el preaviso de reducción del flujo de financiación, la denominada «Información Financiera-PYME» o la revisión de la normativa sobre sociedades de garantía recíproca.

En un segundo momento, nos centraremos en las medidas destinadas a potenciar el uso del mercado de capitales como fuente de financiación. En este sentido, analizaremos el nuevo régimen de titulizaciones aplicable en España para luego detenernos en la novedosa regulación del crowdfunding de corte financiero que introduce esta ley. Finalmente veremos otras cuestiones relativas a la emisión de obligaciones y la negociación en sistemas multilaterales de negociación.

\section{Mejoras de la financiación bancaria a las pequeñas y medianas empresas}

Como se ha comentado, la ley estudiada en este artículo contiene una serie de medidas destinadas a mejorar algunos aspectos de la financiación bancaria.

1 Accesible en http://www.boe.es/boe/dias/2015/04/28/pdfs/BOEA-2015-4607.pdf

2 Las opiniones expresadas en este artículo corresponden exclusivamente a sus autores y no necesariamente representan la opinión de la Secretaría General del Tesoro y Política Financiera.

\subsection{Preaviso e «Información Financiera- PYME»}

El capítulo I de la Ley 5/2015, de 27 de abril, exige a las entidades de crédito ofrecer un preaviso de al menos tres meses a las pymes cuya financiación vaya a ser cancelada o reducida en una cuantía igual o superior al 35 por 100 . Se trata de facilitar que los prestatarios puedan reorientar sus fuentes de financiación antes de que se cierre aquella provista por la entidad de crédito. De esta forma se les proporciona un margen de tiempo muy útil para acudir a otras entidades, a otras fuentes no bancarias de financiación o, llegado el caso, para llevar a cabo un desapalancamiento ordenado que no comprometa la viabilidad de la empresa.

Con esta medida, inspirada en iniciativas de normativas francesas, la financiación bancaria se hace más estable evitando que los problemas de liquidez sobrevenidos por la reducción en el crédito bancario desemboquen en problemas de solvencia. Debe reseñarse, no obstante, que la propia ley exime de la necesidad de preaviso cuando las reducciones en el flujo de financiación derivan de circunstancias como la declaración de concurso del prestatario o la resolución del contrato por incumplimiento de la pyme, casos en los que la reducción en el flujo va más allá de una mera decisión de política comercial.

Como medida complementaria al preaviso, la entidad de crédito deberá además proporcionar gratuitamente a la pyme su «Información Financiera-PYME». Este documento contiene la información que de ella hubiere recabado históricamente la entidad de crédito relativa a su flujo de financiación e incluirá, entre otros, su historial crediticio, una relación cronológica de las obligaciones impagadas o la declaración expresa de que la pyme ha cumplido íntegramente con sus obligaciones, extracto de los movimientos realizados durante el último año y la calificación del riesgo de la pyme, de acuerdo con el contenido y el formato que a estos efectos determine el $D$ 
Banco de España. De esta forma, se resuelven parcialmente las tradicionales asimetrías informativas que existen en el ámbito bancario y que dificultan el acceso al crédito.

La combinación de preaviso e Información Financiera-PYME, puede ser muy efectiva para facilitar que las pymes superen reducciones bruscas en el crédito bancario disponible. En efecto, mientras que el preaviso proporciona un tiempo valioso para buscar alternativas o realizar ajustes, la Información Financiera-PYME le permite probar, frente a otras entidades de crédito, su trayectoria de pagos, con lo que podrá capitalizar un historial de cumplimiento sistemático y ordenado de sus obligaciones.

Finalmente, debe reseñarse que esta información también está disponible para las pymes que, sin sufrir reducción en el flujo de financiación, soliciten su Información Financiera-PYME. No obstante, en este caso, la entidad de crédito no está obligada a realizarlo gratuitamente. En cualquier caso, la mencionada información sigue teniendo utilidad ya que le permitirá negociar, con otros proveedores de fondos, condiciones más favorables.

\subsection{Sociedades de Garantía Recíproca}

Las Sociedades de Garantía Recíproca (SGR), cuyo objeto es ofrecer garantías personales, normalmente en forma de avales, a proyectos empresariales viables que debido a su dimensión o características, encuentran dificultades de acceso a la financiación, han jugado un papel fundamental en la facilitación del acceso a la financiación bancaria para las pymes. Desde su constitución y hasta el 31 de diciembre de 2014, las SGR han avalado un total de 28.512 millones de euros y han apoyado 1.138.414 empleos. Estas cifras han sido posibles, además, gracias a la actuación de la Compañía Española de Reafianzamiento (CERSA), que otorga cobertura parcial de los riesgos asumidos por las SGR con las empresas, reforzando así su solvencia y facilitado su acceso a la financiación.
A pesar de las cifras anteriormente citadas, el sector de las SGR presentaba algunos obstáculos que podían ser claramente superados a través de una mejora de su marco regulatorio. Así, con el fin de fomentar procesos de fusión entre las distintas SGR y acabar con su tradicional atomización, la Ley 14/2013, de 27 de septiembre, de apoyo a los emprendedores y su internacionalización, aumentó el capital social y los recursos propios mínimos exigibles a las SGR hasta los 10 y 15 millones de euros respectivamente.

Por su lado, la Ley 5/2015, de 27 de septiembre, ha continuado con el impulso reformista en el sector de las SGR, mejorando la figura del reaval concedida por la sociedad de reafianzamiento CERSA. De este modo, se permite la activación de este reaval con el simple requerimiento de pago por la entidad de crédito a la empresa, sin necesidad de esperar al requerimiento e incumplimiento por la SGR, lo que sin lugar a dudas permitirá una mejor valoración de esta garantía por las entidades de crédito, redundando así en un mejor acceso y un abaratamiento del crédito para las pymes. Adicionalmente, la Ley 5/2015, de 27 de septiembre, contribuye a la profesionalización y mejor funcionamiento de las SGR, aplicando a sus directivos los requisitos de honorabilidad, conocimiento y experiencia propios de las entidades de crédito.

\section{Mejoras en el acceso a la financiación no bancaria}

Una vez analizados los elementos de la Ley $5 / 2015$, de 27 de abril, que inciden sobre la financiación bancaria podemos ver ahora aquellos que buscan potenciar la provisión directa de fondos por el mercado.

\subsection{Revisión de la regulación de las titulizaciones en España}

La titulización es una técnica financiera consistente en la movilización de activos (habitualmente derechos de crédito) de los balances de $\triangleright$ 
empresas financieras y no financieras que permite captar financiación y promover la transferencia de riesgos de la empresa que cede los activos. Aunque, en teoría, accesible tanto a empresas financieras como no financieras, en la práctica son muy pocas las empresas no financieras que han empleado esta fuente de financiación, quedando la misma reservada fundamentalmente a las entidades de crédito.

Hasta el año 2007, las titulizaciones supusieron una importante fuente de financiación para las entidades de crédito españolas. En 2007 se emitieron en España 141.627 millones de euros en bonos de titulización, siendo el segundo país europeo con mayor volumen de emisiones de este tipo de activos, por detrás del Reino Unido. No obstante, el mal comportamiento de los activos de titulización en Estados Unidos, así como las crecientes tensiones financieras experimentadas por los emisores españoles, redujeron notablemente las emisiones de este tipo de activos, que tocaron suelo en 2012, con emisiones por valor de 23.800 millones de euros.

Entre 2000 y 2012, las titulizaciones americanas presentaron pérdidas del 12 por 100 frente a las titulizaciones europeas, que prácticamente no mostraron pérdidas. A pesar de estas marcadas diferencias, en 2013, en Estados Unidos, se registraron emisiones de bonos de titulización por valor de 2,2 billones de dólares, frente a los 174.000 millones de euros en la Unión Europea.

Dado este injustificado desarrollo de los acontecimientos, la Ley $5 / 2015$, de 27 de septiembre, incluye un nuevo marco regulatorio de las titulizaciones, con el objetivo de fomentar las titulizaciones simples y transparentes y de aproximar nuestro régimen jurídico al de otras jurisdicciones europeas más avanzadas en la materia.

Con el fin de simplificar esta forma de financiación, la Ley 5/2015, de 27 de septiembre, unifica toda la normativa española de titulizaciones, hasta el momento dispersa en diversos cuerpos normativos, en una sola ley. Además, se elimina la distinción tradicional entre los Fondos de Titulización
Hipotecaria y los Fondos de Titulización de Activos, creando una única categoría de fondos de titulización.

En el ámbito de la transparencia, se fortalecen sustantivamente las exigencias, estableciendo la necesidad de que las sociedades gestoras de fondos de titulización publiquen la escritura de constitución del fondo, el folleto de emisión y los informes anuales y trimestrales en sus páginas web. Por otro lado, se permite que los fondos de titulización inscriban, en los registros públicos, la propiedad y demás derechos reales que adquieran, lo que redundará positivamente en la transparencia de su operativa. Además, la nueva norma contempla la posibilidad de que se regule en la escritura de constitución del fondo la creación, atribuciones y régimen de mayorías de la junta de acreedores, previendo así que los inversores expresen su criterio acerca de las distintas situaciones que puedan presentarse. Por último, la nueva ley recoge un régimen sancionador específico para la actividad titulizadora, lo que permitirá que los agentes operen en condiciones de mayor seguridad jurídica.

La Ley 5/2015, de 27 de septiembre, adopta diversas medidas para aproximar nuestro régimen jurídico al de otras jurisdicciones europeas, modificando la regulación que afecta a los activos, a los pasivos y a la estructura y funcionamiento de los fondos de titulización.

En lo referente a los activos de los fondos de titulización, se flexibilizan los mecanismos de adquisición de los activos, de tal manera que ya no sólo podrán adquirirse vía cesión, sino también mediante suscripción, permitiendo así a los fondos acudir al mercado primario de valores.

En cuanto a los pasivos, se elimina la exigencia de que al menos un 50 por 100 de los pasivos de los fondos de titulización deban ser valores, pudiendo a partir de ahora consistir en créditos exclusivamente. También en el ámbito de los pasivos destaca, en línea con el mandato europeo de limitar la dependencia de las agencias de calificación crediticia, la eliminación de la exigencia de que los bonos de titulización tengan que ser objeto de calificación crediticia. 
En relación con la estructura y funcionamiento de los fondos de titulización, habría que destacar la posibilidad de que los fondos de titulización garanticen pasivos emitidos por terceros, lo que permitirá replicar interesantes estructuras de bonos garantizados emitidos con éxito en otras jurisdicciones. Además, se regula expresamente la posibilidad de constituir fondos de titulización con compartimentos y desaparece la prohibición de que el cedente pueda garantizar el buen fin de la operación. Asimismo, la norma reconoce expresamente la posibilidad de crear fondos de titulización de gestión activa, lo que permitirá la modificación de los elementos patrimoniales del activo del fondo con el fin de maximizar la rentabilidad y garantizar la calidad de los activos. Por último, se otorga un período de hasta 4 meses a los fondos de titulización cerrados para incorporar activos hasta un máximo prefijado, lo que permitirá una mejor selección de los activos a incorporar y redundará positivamente en la calidad de los pasivos del fondo de titulización.

Si bien la adopción de un nuevo régimen jurídico nacional en materia de titulizaciones supone un importante avance para el sector, las autoridades europeas están trabajando paralelamente en otras iniciativas que permitan revitalizar esta forma de financiación. En este sentido, cabría destacar la publicación por el Banco Central Europeo y el Banco de Inglaterra de sendos documentos en abril y mayo de 2014 en los que insistían en la importancia de promover estructuras de titulización simples, transparentes y de calidad para reactivar la financiación de la economía europea. Asimismo, los desarrollos técnicos de la normativa bancaria han determinado ya los criterios que han de cumplir los activos de titulización para ser calificados como activos líquidos a efectos de la ratio de cobertura de liquidez. Por último, en el marco del desarrollo de la Unión de los Mercados de Capitales, la Comisión Europea ha señalado como puna prioridad el establecimiento de criterios homogéneos que permitan diferenciar y revitalizar las titulizaciones europeas de alta calidad.

\subsection{La regulación del crowdfunding}

El título V de la Ley 5/2015, de 27 de abril, establece por primera vez en España un régimen jurídico para el fenómeno conocido como crowdfunding. Esta actividad es difusa e incluye muchas variantes. En general se suele dividir en 4 grandes tipos, a saber: recompensas, donaciones, préstamos y emisión de valores. La norma que nos ocupa se ha querido centrar en las dos últimas modalidades, es decir, en el crowdfunding con componente financiero ya que es en este donde están presentes los elementos más urgentes que requieren de una actuación regulatoria, como son el fomento de una nueva fuente de financiación para la economía y la protección proporcionada de las legítimas aspiraciones de los inversores de obtener retorno económico.

El crowdfunding financiero viene a completar, y en ocasiones sustituir, un eslabón en la cadena de financiación de las empresas y los particulares. Las plataformas ofrecen una alternativa frente a un crédito bancario que suele ser más caro, o bien directamente inaccesible. Mediante esta fuente alternativa de financiación se puede proveer de fondos a aquellos proyectos que presentan un mayor riesgo pero también mayor potencial, tanto de corte empresarial como de consumo.

Como señala la exposición de motivos de la Ley $5 / 2015$, de 27 de septiembre, y tal y como ha ocurrido en otros países, la regulación de este fenómeno busca promover el crowdfunding como una posibilidad más dentro de la oferta de fuentes de financiación. Para ello, se ha considerado fundamental proporcionar una cobertura jurídica clara a estas actividades. Procede desligarla entonces de otras actividades de intermediación financiera que están sometidas a una gran cantidad de normas financieras, tanto nacional como europea, normalmente no adaptadas a un negocio tan incipiente como el crowdfunding. No obstante, como no puede ser de otra forma, la norma sigue los principios esenciales que inspiran la regulación $D$ 
financiera e incluye cautelas básicas para asegurar la protección del inversor. Nótese, sin embargo, que esta protección no puede ser absoluta, en particular en un sector como el crowdfunding que debe considerarse de alto riesgo. De esta forma se ha pretendido llegar a un equilibrio entre el fomento de este sector y la protección del inversor.

Dos son los pilares sobre los que se basa esta regulación y que pasamos a ver a continuación: 1) la consideración del crowdfunding como una actividad regulada diferenciada de otras actividades de corte financiero más tradicionales; y 2) la búsqueda de un nivel de protección del inversor acorde con la naturaleza de este novedoso tipo de financiación.

La norma crea una nueva categoría jurídica de entidad de tipo financiero denominada plataforma de financiación participativa que desarrollará una actividad regulada con reserva de actividad. Estas entidades precisarán para operar de la previa autorización de la Comisión Nacional del Mercado de Valores, con la participación del Banco de España en el proceso de autorización en el caso de plataformas de préstamo y/o cuando la entidad solicite ser autorizada como entidad de pago.

Para ello, se define el crowdfunding como la actividad profesional de puesta en contacto, a través de medios electrónicos, de inversores que esperan un rendimiento a su inversión, con promotores que solicitan financiación. Desde un primer momento destaca el componente financiero de esta actividad a través de la búsqueda de un rendimiento, que queda posteriormente confirmado, señalando que las donaciones y compraventas no entran dentro de su ámbito, lo que permite descartar la aplicación de esta normativa a las otras modalidades típicas de crowdfunding mencionadas.

La financiación debe ser recabada en nombre propio y, en este sentido, la norma evita desde el mismo inicio que se utilicen estas plataformas para sortear el resto de normativa financiera que regula el acceso a los mercados de capitales.
Como complemento a lo anterior, se introducen determinadas limitaciones al tipo de proyectos que pueden ser publicados, con lo que se asegura que los fondos captados sean destinados directamente a financiar la economía real.

De especial importancia es la determinación del ámbito territorial porque, a la vez que se introduce una reserva de actividad para las plataformas autorizadas, se clarifica que dicha reserva no es lógicamente aplicable a las actividades realizadas fuera del territorio nacional. Con ello se asegura que tanto promotores como inversores residentes en España pueden acudir a plataformas de otros Estados y, a su vez, estas plataformas pueden admitir sus proyectos y fondos sin incurrir en riesgo legal, siempre que, eso sí, el inversor o promotor hayan acudido a la plataforma extranjera por iniciativa propia.

La Ley 5/2015, de 27 de abril, establece que la captación de fondos puede realizarse mediante la solicitud de préstamos o la emisión de valores negociables (acciones y obligaciones), cuando esta se realice sin el denominado folleto de emisión, o de participaciones de sociedades de responsabilidad limitada. En este sentido, la norma prevé, en la mayoría de ocasiones, un tratamiento similar con independencia de cuál sea el instrumento de captación de ahorro. Aunque los citados instrumentos son jurídicamente distintos y tienen una regulación sustantiva diferente de derechos y deberes, se puede argumentar que, en términos económicos, no hay grandes diferencias entre los préstamos y las obligaciones, o entre las acciones y las participaciones sociales. En consecuencia, la norma opta por un enfoque transversal. No obstante, la diferente estructura jurídica exigirá de los inversores y promotores una especial cautela a la hora de seleccionar cuál debe ser la forma jurídica de captar financiación. En este sentido, los préstamos son la forma más sencilla de captación de recursos en contraposición con las participaciones en el capital social, en las que la condición de socio en el proyecto implicará inevitablemente una mayor complejidad en el ejercicio de los derechos y deberes de la inversión. 
La norma establece una serie de servicios genéricos que pueden desempeñar las plataformas evitando imponer modelo de negocio alguno. Sin embargo, sí determina una serie de prohibiciones para asegurar que los clientes, inversores y promotores no confunden las plataformas de financiación participativa con empresas autorizadas a prestar servicios de inversión, que están sometidas a una mayor carga regulatoria y mayor nivel de protección. En este sentido, se recuerda la prohibición de llevar a cabo servicios de inversión como la gestión de carteras o de recibir fondos por cuenta de clientes y de realizar servicios de pago sin contar con autorización para los mismos. El régimen jurídico establecido por la norma es, por lo demás, parecido al resto de normativa financiera exigiendo, entre otras cuestiones para su autorización, requisitos financieros, requisitos de honorabilidad y experiencia para los administradores y mecanismos de gestión de conflictos de interés.

La regulación de las plataformas de financiación participativa asegura un nivel suficiente de protección al cliente adaptado a este tipo de inversiones. Para ello, se pretende minimizar el riesgoplataforma al que están sometidos los inversores y promotores. En este sentido, debemos citar las mencionadas prohibiciones como la de gestionar pagos sin la oportuna licencia y el establecimiento de normas de conducta por las que las plataformas están obligadas a actuar de forma diligente, transparente, neutral y en el mejor interés de sus clientes, entendiendo por tales tanto a los inversores como a los promotores de los proyectos.

Pese a las anteriores medidas de protección del inversor, no puede descartarse la posibilidad de que el proyecto en el que un inversor invierte no reporte rendimiento alguno, o incluso que el promotor no utilice los fondos para el destino anunciado. Es decir, los inversores están sometidos al riesgo-promotor y la ley recuerda que los proyectos no son objeto de autorización ni de supervisión por la Comisión Nacional del Mercado de Valores.

No obstante, para mitigar este riesgo, la norma incorpora una serie cautelas. Así, el capítulo IV del título $V$ de la ley establece los requisitos que deben cumplir los proyectos y promotores antes de la publicación de los proyectos en una plataforma de financiación participativa. Cabe reseñar la obligación de que el proyecto cuente con un mínimo de información que permita al inversor formarse una opinión sobre el mismo, la obligación de determinar un objetivo máximo de financiación a captar en un plazo predeterminado, y la existencia de un límite máximo de 2.000 .000 de euros que podrá ser de 5.000 .000 de euros cuando los proyectos se dirijan exclusivamente a inversores acreditados. Aunque se impone un deber de diligencia sobre las plataformas para que se aseguren que los proyectos y promotores cumplen con lo establecido en la ley, la norma recuerda que la responsabilidad de la información de los proyectos recae en última instancia sobre los promotores. Como no puede ser de otra forma, los inversores que son los que en última instancia van a soportar las pérdidas de su inversión, deben extremar la diligencia a la hora de seleccionar los proyectos a los que conceden fondos.

Finalmente, como medida adicional de protección del inversor, la norma segmenta los inversores distinguiendo entre los denominados inversores acreditados que cuentan con mayores conocimientos y capacidad para soportar pérdidas, y los inversores no acreditados, en esencia de carácter minorista. Esta segmentación permite que los inversores de corte más profesional no tengan que estar sometidos al régimen de advertencias previas a la inversión ni, sobre todo, a los límites cuantitativos a la misma, lo que favorece el despegue de las plataformas de financiación participativa. Por contra, los inversores no acreditados, por su carácter minorista, están sometidos a un mayor nivel de protección estableciendo mayores advertencias y límites anuales a la inversión (3.000 euros por proyecto y 10.000 euros en el conjunto de plataformas).

\subsection{Mejora del régimen de emisión de obligaciones}

La Ley 5/2015, de 27 de septiembre, viene a culminar la reforma del régimen de emisión de obligaciones que se había iniciado ya con otras iniciativas como la creación del Mercado Alternativo $D$ 
de Renta Fija (MARF), sistema multilateral de negociación que vio su primera emisión en diciembre de 2013, o la actualización por la Comisión Nacional del Mercado de Valores (CNMV) de las directrices para la emisión de obligaciones y pagarés.

La Ley 5/2015, de 27 de septiembre, permite por primera vez a las sociedades de responsabilidad limitada emitir obligaciones, fuente de financiación que hasta ahora les estaba vedada, abocándolas a depender especialmente de la financiación bancaria. Sin embargo, en general, se puede argumentar que la garantía patrimonial ofrecida por una sociedad de responsabilidad limitada no es comparable a la proporcionada por sociedades anónimas, cuyo capital social mínimo es más elevado. Por ello, el legislador ha optado por limitar la capacidad emisora de las sociedades limitadas, fijándola como máximo en el doble de sus recursos propios, salvo que la emisión esté garantizada con hipoteca, con prenda de valores, con garantía pública o con un aval solidario de entidad de crédito.

En lo que a las sociedades anónimas respecta, se elimina el límite cuantitativo a sus emisiones que hasta la entrada en vigor de esta ley se cifraba en el importe resultante de la suma de su capital social desembolsado, las reservas del último balance y las cuentas de regularización. Con ello se potencia esta fuente de financiación vía mercado de capitales.

Adicionalmente, la Ley 5/2015, de 27 de septiembre, introduce diversas medidas tendentes a agilizar el proceso de emisión de obligaciones. En este sentido, cabría destacar la eliminación de la exigencia de inscribir en el Registro Mercantil la escritura pública en la que se formaliza la emisión de obligaciones, así como su correspondiente publicación en el Boletín Oficial del Registro Mercantil (BORME). Además, se atribuye al consejo de administración, en lugar de a la junta general de accionistas, la competencia de aprobar la emisión, lo que introducirá mayor dinamismo en el acceso de las empresas a los mercados.

Por último, la nueva norma clarifica los supuestos en los que resultará necesaria la constitución de un sindicato de obligacionistas bajo ley española, acabando así con situaciones del pasado, en las que una empresa española que quisiera emitir obligaciones en el extranjero se veía obligada a cumplir simultáneamente con la normativa española y con la del país de la emisión en materia de protección de inversores.

Todo ello redunda en que la emisión de obligaciones se posicione como alternativa viable a la financiación bancaria para las pymes.

\subsection{Potenciar el funcionamiento de los sistemas multilaterales de negociación}

Dentro del título IV de mejoras en el acceso y funcionamiento de los mercados de capitales, se incluyen medidas para facilitar el tránsito desde los sistemas multilaterales de negociación hacia los mercados secundarios oficiales.

La admisión a negociación en un mercado secundario oficial suele ser el objetivo de muchas empresas ya que implica una visibilidad incomparable e incrementa su capacidad para captar recursos tanto ajenos como propios. No obstante, esta admisión a negociación conlleva también el sometimiento por parte de las empresas a una gran cantidad de normativa financiera que pretende asegurar el comportamiento eficiente del mercado, un buen gobierno corporativo y, en suma, la protección del inversor. Para facilitar el paso desde los sistemas multilaterales de negociación a los mercados secundarios oficiales, la Ley 5/2015, de 27 de abril exime durante dos años a las empresas de cumplir con la obligación de realizar las declaraciones intermedias de gestión y el denominado segundo informe semestral.

Adicionalmente, se introducen medidas para asegurar el buen funcionamiento de los sistemas multilaterales de negociación reforzando las herramientas de supervisión. En esta misma línea, se establece también un umbral de cotización a partir del cual las empresas negociadas en los sistemas multilaterales de negociación deben solicitar la admisión en un mercado secundario oficial de forma que se les aplicarán las exigencias propias de los mercados secundarios oficiales, más $D$ 
estrictas, como por ejemplo, las concernientes al gobierno corporativo.

\section{Conclusión}

A lo largo de este artículo hemos repasado las principales novedades que introduce la Ley $5 / 2015$, de 27 de abril, de fomento de la financiación empresarial. Aunque esta ley entró en vigor al día siguiente de su publicación, debemos esperar todavía a que las medidas en ella previstas se empiecen a aplicar, ya que se han establecido periodos transitorios para permitir la adaptación del mercado a sus disposiciones.

La ley incorpora además otras medidas que son también de interés como es el establecimiento del nuevo régimen jurídico de los establecimientos financieros de crédito con el que se moderniza la regulación de este tipo de entidades tan relevantes en la financiación al consumo minorista. Por otro lado, la ley también recoge un conjunto de modificaciones a la Ley del Mercado de Valores con objeto de reforzar la capacidad de supervisión de la CNMV.

Además, debe resaltarse que esta ley viene a complementar otras iniciativas destinadas a promover la financiación parabancaria como es la reforma del régimen del capital riesgo, a través de la Ley 22/2014 que, entre otras cuestiones, ha flexibilizado la normativa para favorecer que haya una mayor participación a través de deuda.

La necesidad de potenciar la financiación de las empresas (y con el crowdfunding también de los hogares) vía mercados de capitales es un pensamiento compartido por nuestros socios europeos. En este sentido, debe destacarse la reciente iniciativa de la Comisión Europea denominada Unión del Mercado de Capitales. Por tanto, podría argumentarse que España ha sido pionera en una nueva ola de medidas para fomentar el acceso a la financiación alternativa, lo que nos sitúa en una posición privilegiada para contribuir con nuestra experiencia al desarrollo del nuevo proyecto europeo.

\section{Bibliografía}

[1] ÁLVAREZ-CIENFUEGOS RICO, F. (2015). «EI impulso normativo de las formas de financiación alternativa en la economía española». Revista Iberoamericana del Mercado de Valores. Disponible en:

http://www.rimv.org/es/revista-2015-julio/articulo/ resumen/1010/el-impulso-normativo-de-lasformas-de-financiacion-alternativa-en-laeconomia-espanola

[2] ARNAL MARTÍNEZ, J. y RODRÍGUEZ FERNÁNDEZ, S. (2015). «Modificaciones y posibilidades legislativas recientes para impulsar la financiación a la empresa». Anuario sobre renta fija y financiación alternativa IEB-Axesor 2015.

[3] BANCO CENTRAL EUROPEO y BANCO DE INGLATERRA (2014). The Case for a Better functioning Securitisation Market in the European Union. A Discussion Paper. ECB Disponible en:

https://www.ecb.europa.eu/pub/pdf/other/ecbboe_case_better_functioning_securitisation_ marketen.pdf

[4] BANCO DE ESPAÑA (varios años). Indicadores económicos e Informe de Estabilidad Financiera. Disponible en: http://www.bde.es/bde/es/secciones/ informes/

[5] COMISIÓN EUROPEA (2015). Libro Verde. Construir una Unión de los Mercados de Capitales. Bruselas. Disponible en:

http://eur-lex.europa.eu/legal-content/ ES/TXT/PDF/?uri=COM:2015:63:FIN\&from=ES

[6] COMISIÓN NACIONAL DEL MERCADO DE VALORES (2015). Estadísticas del mercado primario de valores. Disponible en:

http://www.cnmv.es/portal/Publicaciones/ estadisticas.aspx

[7] COMISIÓN NACIONAL DEL MERCADO DE VALORES (2013). Guía de renta fija: Criterios, procedimientos y documentación requerida para la tramitación de operaciones de renta fija. Disponible en:

http://www.cnmv.es/DocPortal/GUIAS_Perfil/ guia_renta_fija.pdf

[8] COMPAÑÍA ESPAÑOLA DE REAFIANZAMIENTO (2015). Datos del sector de las sociedades de garantía recíproca. Disponible en:

http://www.cersa-minetur.es/seccion/el-sistemade-garantia/principales-cifras-1

[9] REVELLO DE TORO CABELLO, J.M. (2013). Manual de Corporate Finance y Banca de Inversión, Delta Publicaciones. 
